\title{
Numerical and experimental investigation on the effect of retrograde vaporization on fines migration and drift in porous oil reservoir: roles of phase change heat transfer and saturation
}

\author{
B. Kanimozhi ${ }^{1}$ (D) Jaya Prakash ${ }^{1} \cdot$ R. Venkat Pranesh ${ }^{2} \cdot$ S. Mahalingam ${ }^{3}$
}

Received: 19 January 2019 / Accepted: 11 May 2019 / Published online: 21 May 2019

(c) The Author(s) 2019

\begin{abstract}
Retrograde vaporization effects on oil production are nearly unprecedented to reservoir engineering community, and its relation to formation damage should be explored. For this purpose, this paper elucidates the importance and role of this phenomenon and its phase change heat transfer (PCHT) on fines migration and subsequent, permeability damage in porous rocks bearing oil and gas. Initially, a fine particle energy conversion equation was successfully acquired by combining fine particle mass balance and general energy equations. Moreover, the computational fluid dynamic model (CFD) was adopted for performing numerical modeling. A 2D CFD model using FEA-Comsol 5.0 version was used to simulate the retrograde vaporization of reservoir fluids. Pore walls are designed as non-adiabatic, and therefore, a modified Dittus-Boelter mass transfer model is provided for a fine particle detachment under PCHT. Hence, from the simulation results it was observed that there is a high degree of heat release during reservoir fluid phase change that is from oil to gas for decreasing pressure and increasing saturation time. This heat transfer from the oil and gas influxes contributes in the expulsion and migration of in situ fines in porous media. Also, an increasing rate of enthalpy was achieved that produces a non-isentropic flow, which is required to mobilize the fines in porous medium, and a satisfactory phase transition simulation outputs were obtained and presented as well. Altogether, these factors play a significant role in the fine particle eviction from the pore chamber, thereby plugging in the pore throat and consequently, decreasing the well productivity during transient flow.
\end{abstract}

Keywords Retrograde vaporization · Fines migration · Phase change heat transfer · Arc length · Enthalpy

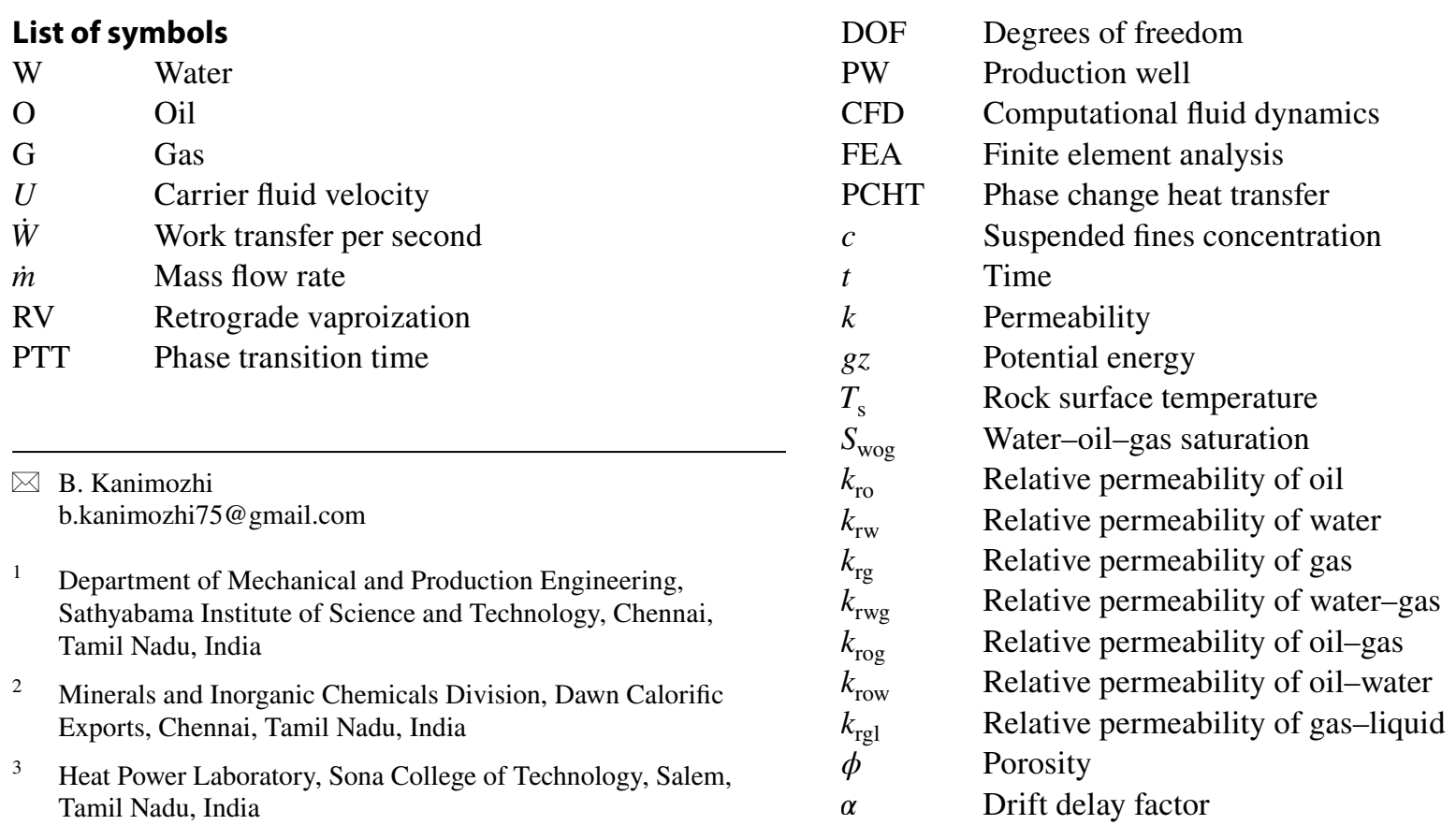

Tamil Nadu, India 


$\begin{array}{ll}\sigma_{\mathrm{a}} & \text { Concentration of attached particles } \\ \sigma_{s} & \text { Concentration of strained particles } \\ \frac{P}{\rho} & \text { Flow energy } \\ \frac{v^{2}}{2} & \text { Kinetic energy } \\ \nabla(s+\gamma) & \begin{array}{l}\text { Overall change in entropy }(s) \text { and surface } \\ \text { energy }(\gamma) \text { in porous media }\end{array}\end{array}$

\section{Introduction}

Oil reservoirs are frequently prone to permeability damage and well productivity decline, especially due to natural reservoir fines migration (Chequer et al. 2018). Subsequently, waterflooding process is employed when the reservoir is at the stage of pressure loss and for efficient oil recovery as well (Alhuraishawy et al. 2018). Even during waterflooding, a huge amount of clay fines are detached from the rock surface and suspended in the permeating fluid, and consequently, transported by the fluid in the form of suspension flow. Clay fines mostly consist of kaolinite particles that exist in pore space, and like colloids, generally, fines have a size of the order of $1 \mu \mathrm{m}$ and a net surface charge (Raha et al. 2007). Fines migration during waterflooding was given much importance in recent years and with that many analytical models were developed. For many decades, the oil and gas industry employs water to mobilize oil in the reservoir during its pressure reduction and a schematic diagram is shown in Fig. 1. At the same time, the water salinity and chemistry play a vital role in detachment and the mobilization of reservoir fines, and the knowledge of its literature studies is crucial for formation damage quantification and mitigation.
In contrast, there were also reports on improved oil recovery by fines assisted waterflooding (Hussain et al. 2013). Zeinijahromi et al. (2016) investigated the fines migration effects on relative permeability of oil-water phase during two-phase flow in porous media. The effect of fines transport on oil recovery and relative permeability during two-phase flow is still incoherent. The formation of water salinity can vary from that of the injected water and subsequently affects the relative permeability. The authors conducted coreflood with piecewise constant declining of water salinity without oil for the identification of fines mobilization. For recovery evaluation exclusive of ion exchange process, the oil is displaced with formation of water in sister cores and oil is displaced again by water with different salinity after restoration. The relative permeability was stated by modified JBN method for coreflood test with the migration of fines, and it was observed at high saturations that water relative permeability curve declines due to fines migration and straining which results in water permeability deterioration.

Zeinijahromi et al. (2011) analyzed the induced formation damage effects on water cut during waterflooding. It was revealed from various studies that there was fines migration during coreflood test with changes in water chemistry and temperature. In this case, the authors have given importance to mobility control during waterflooding. In earlier research, the particle detachment model was combined with a Dietz model for a layer cake reservoir under waterflooding with constant injection and production rate to examine the fines migration effect and induced permeability reduction in the sweep efficiency of the reservoir. They developed and extended an analytical model to waterflooding with pressure drop between injection and production wells.
Fig. 1 Typical process of waterflooding in an oil reservoir (Tistory 2018)

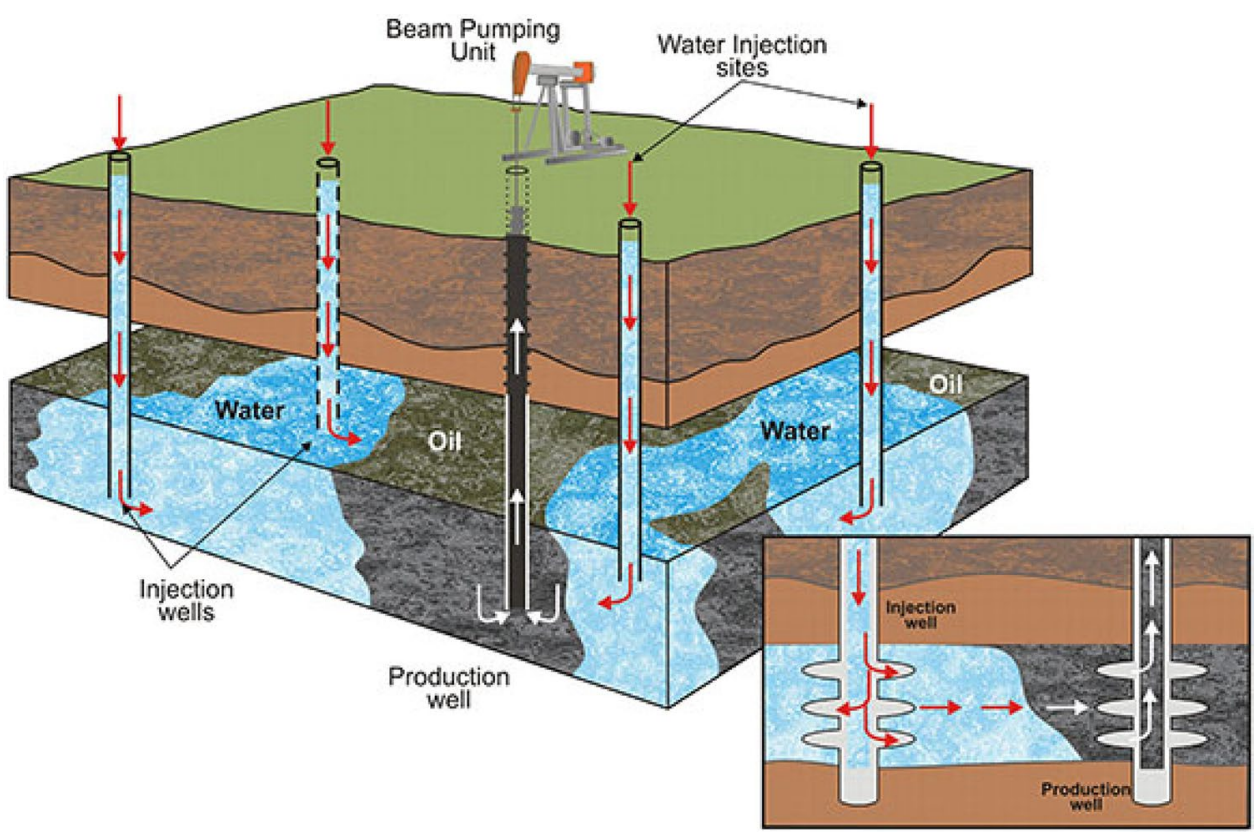


Figure 2 shows the oil and gas reservoir fines behavior in a single-pore chamber system. It can be seen from the above figure that the fine particle is under the influence of four forces namely lift, electrostatic, gravity, and drag which are denoted as $F_{\mathrm{l}}, F_{\mathrm{e}}, F_{\mathrm{g}}$, and $F_{\mathrm{d}}$, respectively. These four major forces are determined by the torque balance that in turn constitutes the mechanical equilibrium of attached particles. Fines adhering to the surfaces of the pore of the relatively higher porosity and permeability porous formations can be released by colloidal, hydrodynamic, and thermodynamic forces, and these forms of induced fines are identified to be a more common occurrence in sandstone formations, especially water-wet rocks. These particles, releasing from and adhering to the pore surface, avail the potential energies of London-van der Waals attraction and electrical double layer repulsion, respectively (Khilar and Fogler 1998). The former force is the attraction energy at the interface between the fine particle and pore wall and contributes to its attachment strength. The latter force describes the formation of electric double layers at the surface of the pore and around the fine particle which overlap and expand. This process repels the fines from the surfaces and induces the migration in the porous medium.

During water-oil mobilization in porous medium, there will be a decrease in relative permeability. While well is produced, there is an observation of low pressure near the region of wellbore. Consequently, this pressure fall can lead to gas release (Robinson 1993). So, there is a creation of three fluids in the porous rocks which enhances the fluid saturation and affects the surface energy besides fluid recovery as well, due to permeability blockage. In due course, the reservoir pressure will deteriorate further and flow efficiency of the primary fluid will be drastically reduced. Therefore, this paper aims to address the problem of gas release from oil phase, also known as retrograde vaporization (RV) and its effect on in situ fines migration and formation damage. The RV phase diagram is presented in Fig. 3. Additionally, due to the presence of three different fluids having different

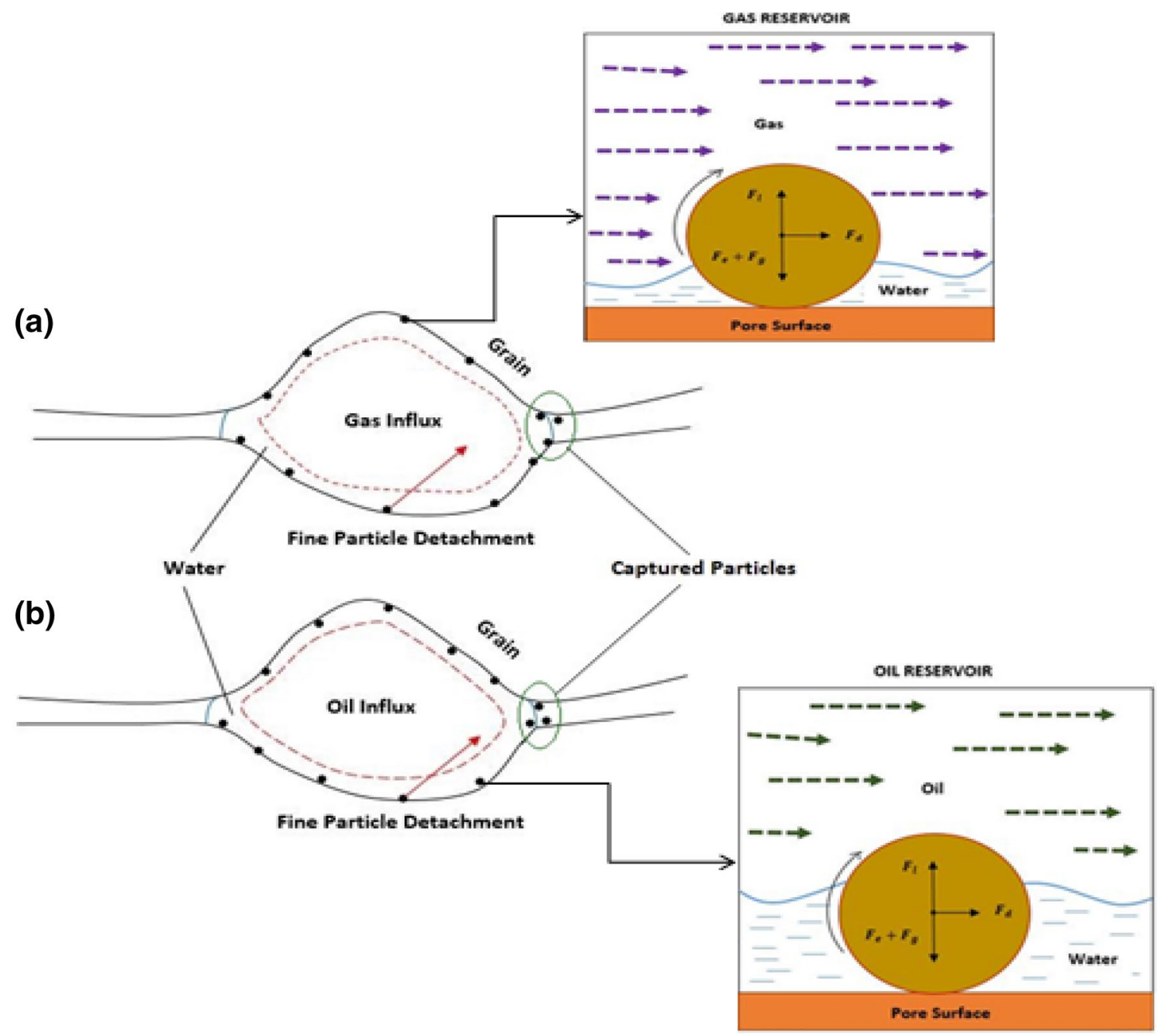

Fig. 2 Fines behavior in single-pore chamber system, a gas reservoir, $\mathbf{b}$ oil reservoir 


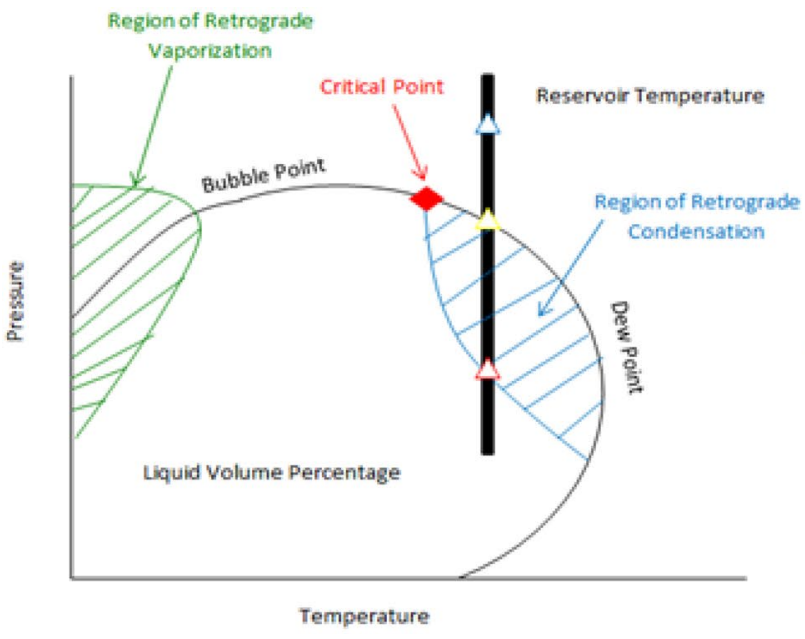

Fig. 3 Phase behavior of retrograde vaporization of an oil reservoir

physical and chemical properties, the suspended fines will drift in the pore space and there will be a slow mobilization due to decrease in its degree of its freedom.

There is also a report of slow transport and fines drift in the porous rocks. For instance, Oliveira et al. (2014) studied this same phenomenon of slow mobilization of reservoir fines in porous media, and specifically, authors have reported from experiments that the speed of mobilized fines drift is considerably lower than the carrier fluid (water) velocity. It should be noticed that this fine drifting can be a sum of different particle micro-motions like rolling over the surface of the rock and sliding in the walls of the rock pores. Hence, it is necessary to investigate the RV effect on the clay fines drift and stagnation in porous media. Most researchers are unaware that the retrograde vaporization and well-known phenomenon in gas reservoirs during pressure drop near wellbore is retrograde condensation, in which, there will be a liquid from the gas phase due to reduction. As a result, the well will experience liquid loading effect and production loss. Likewise, the retrograde vaporization process also has a tremendous effect on the well productivity; the only difference is that this phenomenon will occur in oil reservoirs. To the best of our knowledge, till date this retrograde vaporization process and mechanism in oil reservoirs and its effect on natural reservoir fines have not been addressed yet. Hence, we have conducted mathematical and numerical modeling to understand this phenomena and our modeling results were good.

\section{Modeling methodology}

A multiphysics flow FEA-equipped CFD model with COMSOL 5.0 code was employed to simulate the dynamic vaporization in an oil reservoir. A two-dimensional

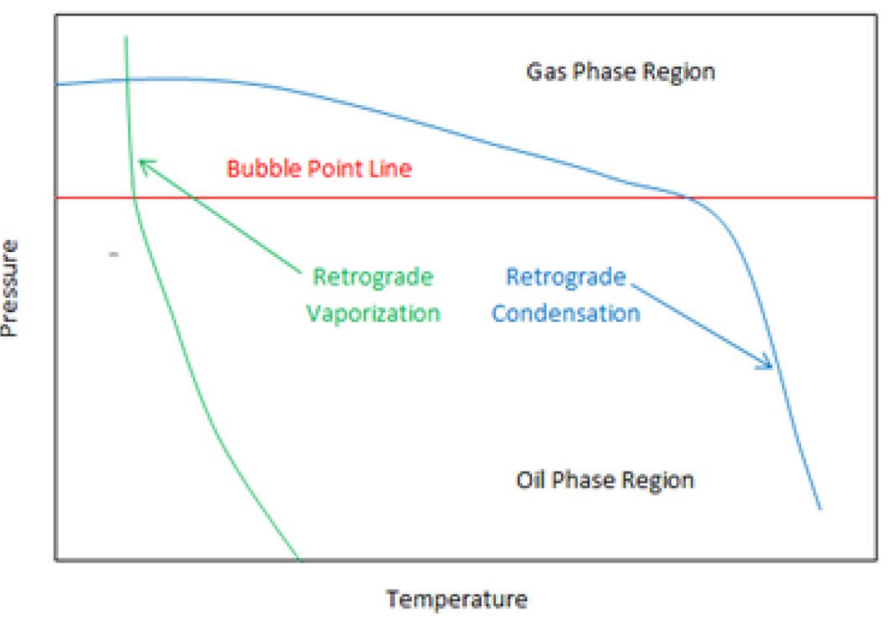

computer-aided design (CAD) geometry model of top view of production well (PW) and reservoir formation has been created with a definite geometry and mesh for the fluid phase change simulation, which is shown in Fig. 4. $T_{\mathrm{s}}$ indicates the surface temperature of the porous formation. Furthermore, $k \frac{\partial T}{\partial x}$ indicates the change in surface thermal conductivity. A framework has been made in such a way that during transient calculations the fluid properties are calculated with the given reservoir temperature at any given time during the iterative calculation. The simulation was carried out in the phase order oil to gas. Water-oil mobilization alters the reservoir pressure and temperature. The latter physical quantity over the pore surface boundary has been mentioned by obtaining a value by an empirical correlation of the Dittus-Boelter Equation. Material properties of the governing equations are embedded as inputs to the solver and to the account or time dependency of the problem as well as for the gas flow and heat transfer in the

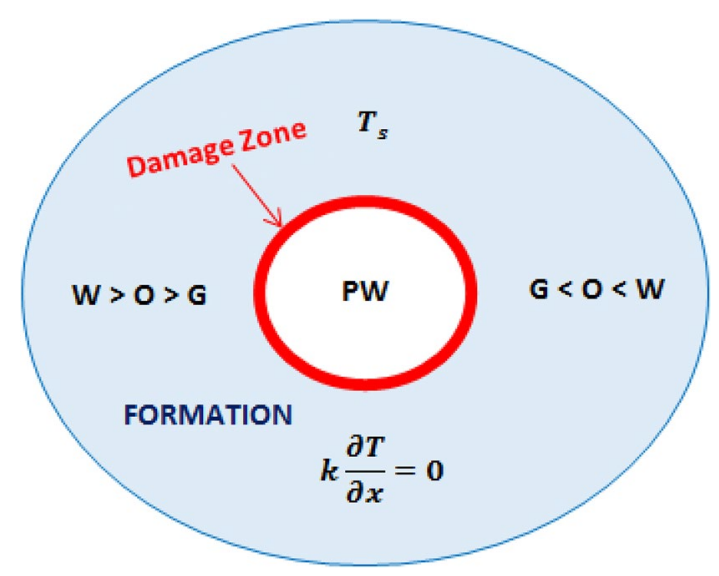

Fig. 4 2D CAD diagram of the top view of production well and reservoir formation 
porous media. A transient analysis under fluid-thermal interaction was used, to solve both Navier-Stokes equation and the energy change equation of the retrograde vapor radiation and conduction.

The energy change in porous media due to fine particle detachment and straining is analyzed in this section. The mass balance equation for suspended, attached and strained particles is mentioned as below (Yang et al. 2016; You et al. 2016):

$\frac{\partial}{\partial t}\left[\phi c+\sigma_{\mathrm{a}}+\sigma_{\mathrm{s}}\right]+\alpha U \frac{\partial c}{\partial t}=0$

where $c, \sigma_{\mathrm{a}}$ and $\sigma_{\mathrm{s}}$ are concentrations of suspended, attached and strained particles, respectively. $i$ is the flow velocity of the carrier fluid that coincides with particle speed and $\alpha$ is a drift delay factor.

The general energy equation, which is obtained through the first law of thermodynamics, is as follows:

$\dot{m}\left(\frac{P}{\rho}+\frac{v^{2}}{2}+g z\right)+\dot{W}=0$

where $\frac{P}{\rho}=$ Flow energy, $\frac{v^{2}}{2}=$ Kinetic energy, $g z=$ Potential energy, $\dot{m}=$ Mass flow rate, $\dot{W}=$ Work transfer per second.

By adding Eqs. 1 and 2, we get:

$\frac{\partial}{\partial t}\left[\phi c+\sigma_{\mathrm{a}}+\sigma_{\mathrm{s}}\right]+\alpha U \frac{\partial c}{\partial t}+\dot{m}\left(\frac{P}{\rho}+\frac{v^{2}}{2}+g z\right)+\dot{W}=0$

The above Eq. (3) can be further written as follows:

$\frac{\partial}{\partial t}\left[\phi c+\sigma_{\mathrm{a}}+\sigma_{\mathrm{s}}\right]+\alpha U \frac{\partial c}{\partial t}+\dot{m}\left(\frac{P}{\rho}+\frac{v^{2}}{2}+g z\right)+\dot{W} \pm \nabla(s+\gamma)=0$

where

- $\nabla(s+\gamma)=$ Overall change in entropy

$(s)$ and surface energy $(\gamma)$ in porous media
- Entropy and surface energy rises during particle detachment from pore wall.

- Entropy and surface energy declines during particle straining in pore throat and also during particle re-attachment.

- The Plus or minus( $( \pm)$ sign indicates increase and decrease in entropy and surface energy.

This explanation for Eqs. 3 and 4 mainly accounts for energy change. The energy change during particle detachment (as a consequence of waterflood and/or high reservoir temperature) and straining can be described by Fig. 5 .

Since Eqs. 3 and 4 consider the variable drift delay factor, we shall take the waterflooding as permeating fluid for fines surface detachment and have divided the porous media into three sections to describe the energy change.

Section 1 During waterflooding (flow in pore space) there is an increase in kinetic energy (including fluid energy) and velocity. Subsequently, there are no production and creation of entropy and new surface. Here the potential energy may remain constant. In this location, there is no presence of fines or if there is a particle, it is firmly attached to the rock surface. Here the particle is not affected during fluid flow velocity.

Section 2 In this location, the fines are detached from the pore wall and after particle detachment there is a production of entropy and surface energy resulting in the creation of new surface. Over here, the detached particles are suspended in water and some particles are strained and plugged in the pore throat. The new surface energy increases the volume of the pore chamber for more fluid storage. In this part, the kinetic energy and velocity of fluid is in equilibrium and there is a rise in potential energy.

Additionally, during particle suspension or suspension flow the particles will undergo a collision with pore walls and as a result the particle will lose its momentum. After losing momentum, it is again reattached to the rock surface and then there is a decline in available entropy and
Fig. 5 Illustration of fine particle energy conversion process in a single-pore chamber system

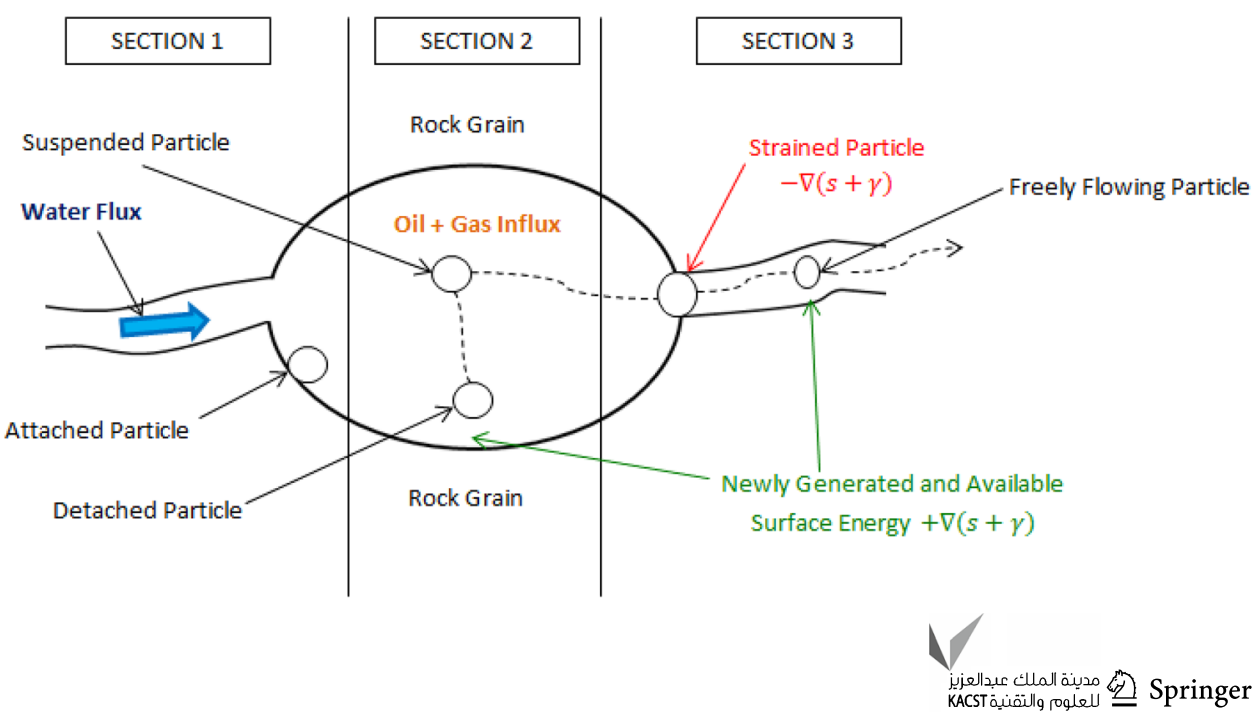


surface energy (Kampel 2007). Furthermore, some particles may undergo a slow mobilization over rock surface, this is because of the decrease in the fluid momentum and internal energy (including enthalpy).

Section 3 At this location, the suspended particles are captured or strained in pore throat. Here there is a loss in entropy and surface energy. Also, the fluid kinetic energy and velocity will exhibit an exponential decline. Mainly at this point, there is a huge amount of potential energy, since the pore chamber is filled with water that is suspended with fines (colloid). It should be noted that already in Sect. 2 there is a creation of new surface space (due to fines detachment).

Collectively or in a straightforward manner, it can be stated or proposed that Eqs. 3 and/or 4 are fines energy conversion equation or in other words, we hereby propose that by adding a general energy equation to fines mass balance equation yields energy conversion equation for fine particles in porous media. It can be clearly seen that two different equations are equated to yield an energy conversion equation for fines transport in porous media.
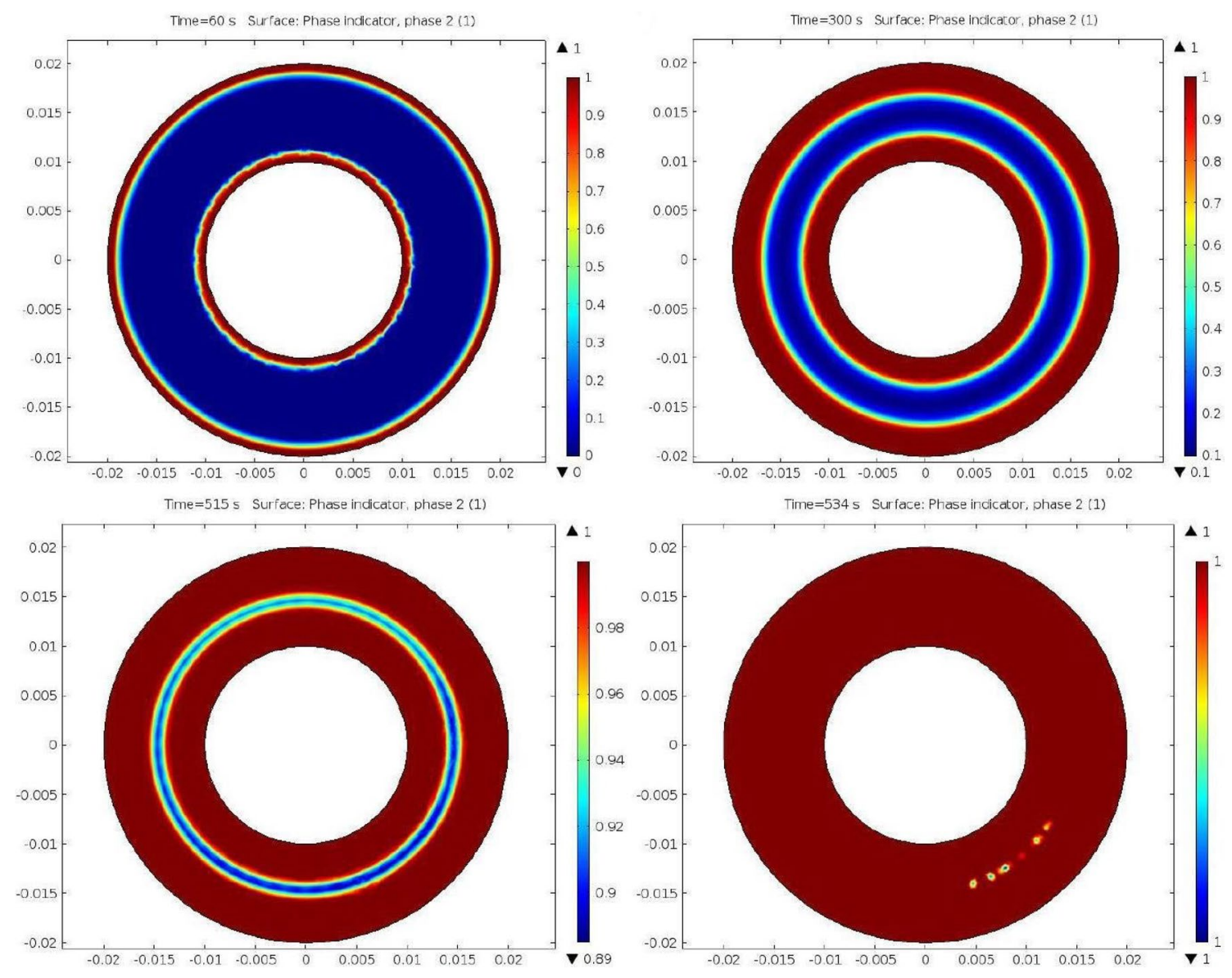

\section{Results and discussions}

This section discusses the CFD modeling results and the curves obtained.

\section{Effect of retrograde vaporization on fines drift in porous rocks}

Figure 6 shows the retrograde vaporization process in the top view for the phase transition times $60 \mathrm{~s}, 300 \mathrm{~s}, 515 \mathrm{~s}$, $534 \mathrm{~s}$. It can be seen from these figures that the blue contour represents the oil phase and maroon indicates the gas phases, and in the center, a white circle indicates the area of the production well. It can be seen from the top left side of Fig. 6 that the reservoir is highly saturated with oil phase with trace amounts of gas phase, but here the phase transition time (PTT) is $60 \mathrm{~s}$. When the time increases to $300 \mathrm{~s}$, the mass of gas phase increases. Further, it increases for $515 \mathrm{~s}$ and $534 \mathrm{~s}$ PTT. But in the latter case the top layer of the reservoir is saturated with the gas phase. It should be noted that at this time, the reservoir porous rocks is dominated by the gas

Fig. 6 Top view of the retrograde vaporization process in an oil reservoir 
phase. However, the readers should not assume that the oil phase is completely diminished. Actually, the simulation was performed in the top view and the gas phase has overtaken the oil phase or in other words, the gas phase has left the oil phase below. Obviously, the density of the gas phase is very low when compared to oil and water phases. Therefore, the gas phase will occupy the top layer of the porous rocks. By taking Fig. $2 b$ into account, we can hereby state a new surface energy is created in the pore chamber during the phase transition time, which the gas phase will occupy the space. Meanwhile, in the middle and bottom regions the surface energy will neither decline nor oscillate due to the presence of oil-water phase.

During the retrograde vaporization process, there will be a high release of heat and will be transferred to the surroundings. This type of heat release during the RV process or during phase transition is called the phase change heat transfer. During this time, the reservoir may experience a higher temperature and it is also possible due to the presence of the gas phase. This high temperature enhances the pore surface thermal conductivity and as a result the electrostatic force, which hold fines on the rock surface, becomes weak. Subsequently, the fines will be detached and suspend in the fluid, and will be transported by the carrier fluid. It is actually complicated to establish a formation damage framework for this fines migration problem.

Before critically analyzing the fines drift mechanism, the readers should be aware that at this time the reservoir is saturated with three phases, namely gas-oil-water in the order top-middle-bottom. It is assumed that during PCHT process itself some amount of reservoir fines are detached and suspended in the oil phase, and during PTT, the suspended fines in the oil phase migrate to the gas phase. The particle is jumping from the liquid to gas phase and also colliding with the upper pore wall. Simultaneously, oil-water phase sloshing may occur in the pore chamber (Abbaspour and Hassanabad 2010; Greenspan 2005). Consequently, there will be a loss in the fluid momentum and surface energy in the lower pore wall as well.

In this case, for fines behavior in three-phase system, we apply a fundamental, applied mechanics concept of degrees of freedom (DOF). It is physics of an object that is capable of moving in a specified direction by considering the surrounding medium into an account. The fines are suspended in the gas-oil phase; the suspended fines will collide the upper and lower pore walls and experience a two degrees of freedom. The speed of upper motion is higher than that of the bottom because of the fluid density. Similarly, the suspended fines in the oil-water phase will collide with the sides of the pore wall, and fines now will experience a 3-4 DOF. The fluid momentum will be reduced terribly due to 1-4 DOF. In order to achieve the 5 DOF that is to flow in porous media, the fine particle and the saturated fluid must be accelerated with hydrodynamic and thermodynamic forces. While the $6 \mathrm{DOF}$ is insignificant in this case, it is not considered in this analysis. Figure 7 indicates a typical fine particle under the dominance of 6 DOF.

Already the porous media were saturated with the three fluid phases, and as a result, the porous media with saturated fluids are undergoing a stagnation stage due to loss in the reservoir pressure. There is a decline in well productivity due to this multi-component three-phase fluid saturation. During this period, the detached fines are not captured nor strained in the pore throat. Here the permeability is not
Fig. 7 A typical fine particle is under the governance of the six degrees of freedom (DOF)

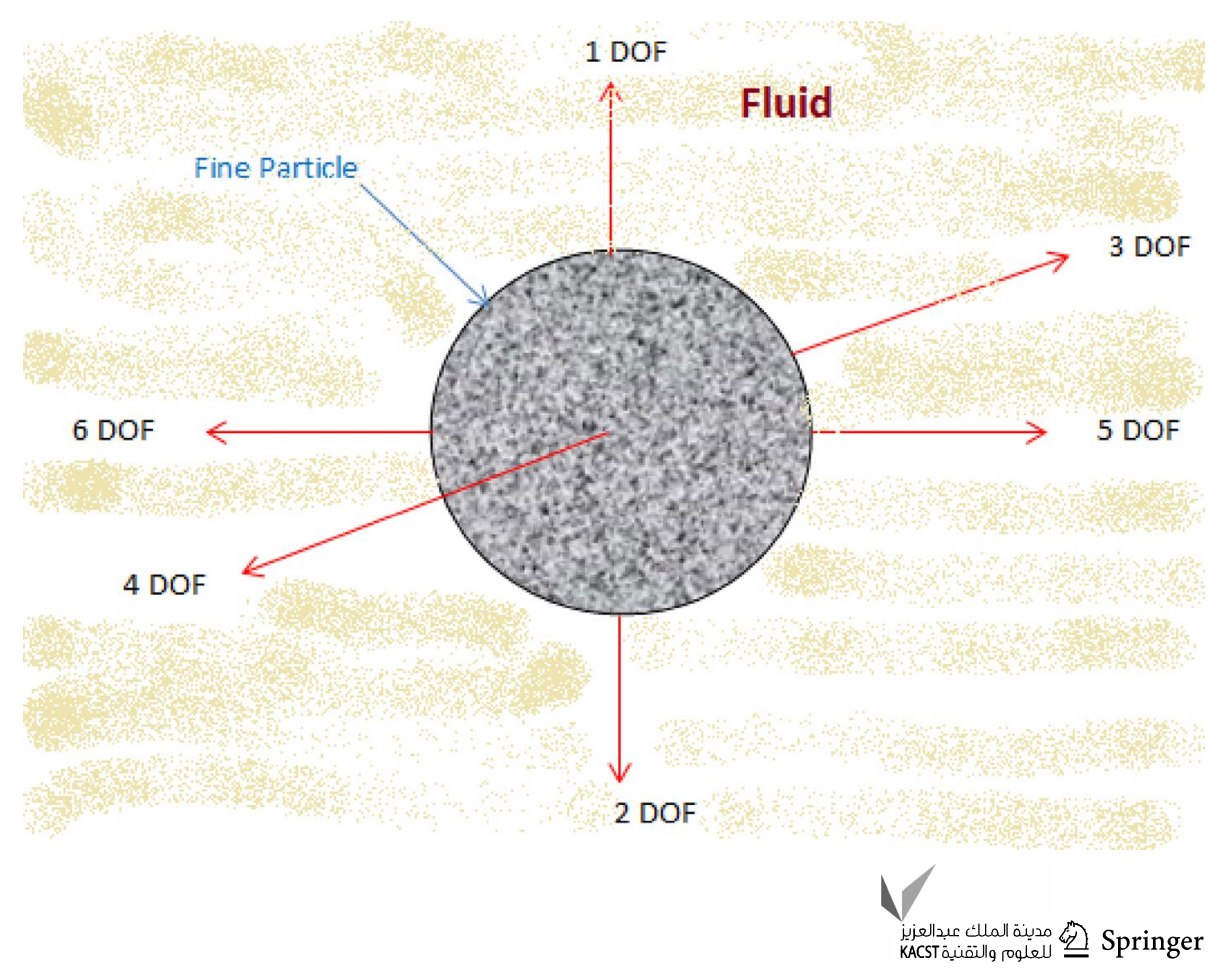


damaged yet and fines will take long time to get trapped in the pore throat. Till that time, the fines will be drifting in the pore wall along with the saturated fluids. Whatsoever, the permeability is going to be damaged, but the thing is that process will take a long time. Figure 8 shows the changes in phase transition intervals, density, and total enthalpy with respect to arc length.

In general, a geometry of porous media can be best represented by a series of a capillary bundle tubes with mixing chamber (Dullien 1992; Santos et al. 2008; Bashtani et al. 2013). It can be clearly seen from Fig. 8 that three physical parameters vary with respect to different capillary bundle tube arc length. It is mathematically defined as the pore chamber of radius $r$ with central angle $\theta$ radians or a portion (length of the arc divided by the radius of the arc) of a pore chamber in which the vaporization has occurred. Actually, it is measuring the RV from a pore point by taking its length, radius and angle into an account. It can be seen from Fig. 8a that there is a high degree of phase transition intervals for increasing arc length. Different curves were obtained, which indicate for various PTT and similar recordings were observed for Fig. 8b, c. A flat upward and inverted " $U$ " shaped curve was acquired, which indicates the feasibility of non-Newtonian flow in the porous medium due to differences in the saturated fluid physical properties. Figure 9 shows the variation of total enthalpy with respect to increasing temperatures.

It can be observed from Fig. 9 that for all fluid phase transition times the enthalpy of the reservoir fluids increases rapidly for increasing temperature. It can be viewed from this figure that in beginning the enthalpy release rate is sluggish and after $30^{\circ} \mathrm{C}$ there is a high degree of enthalpy release for all phase transition times and after that it stabilizes steadily.

This energy release will generate a non-isentropic flow, which also detach the fines from pore surface. The higher enthalpy rate was observed for $500 \mathrm{~s}$ and $540 \mathrm{~s}$ phase transition times. Figure 10 shows the variation of total enthalpy with respect to increase in temperature, specifically
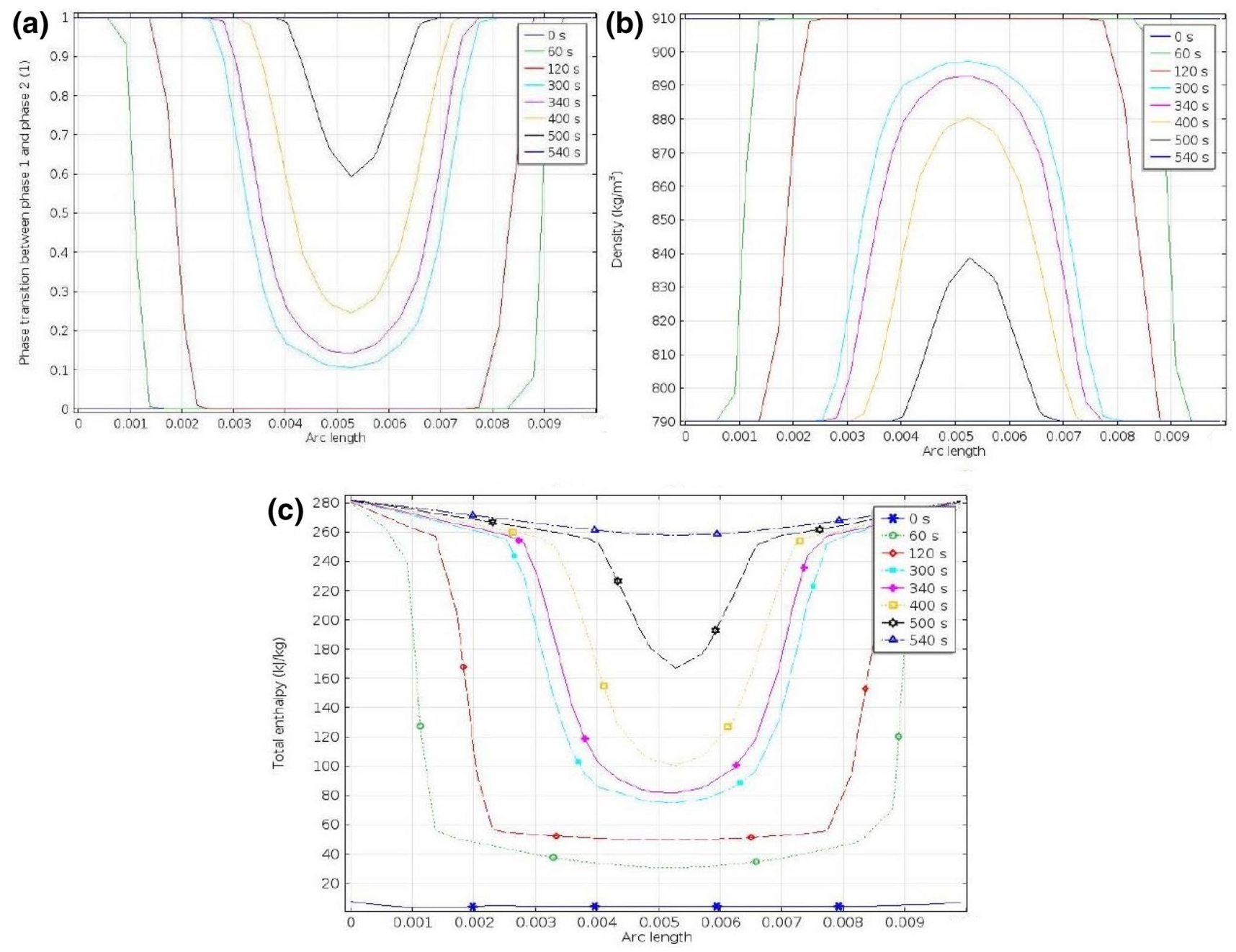

Fig. 8 Physical parameter variations with respect to arc length, a phase transition intervals, $\mathbf{b}$ density, and $\mathbf{c}$ total enthalpy 


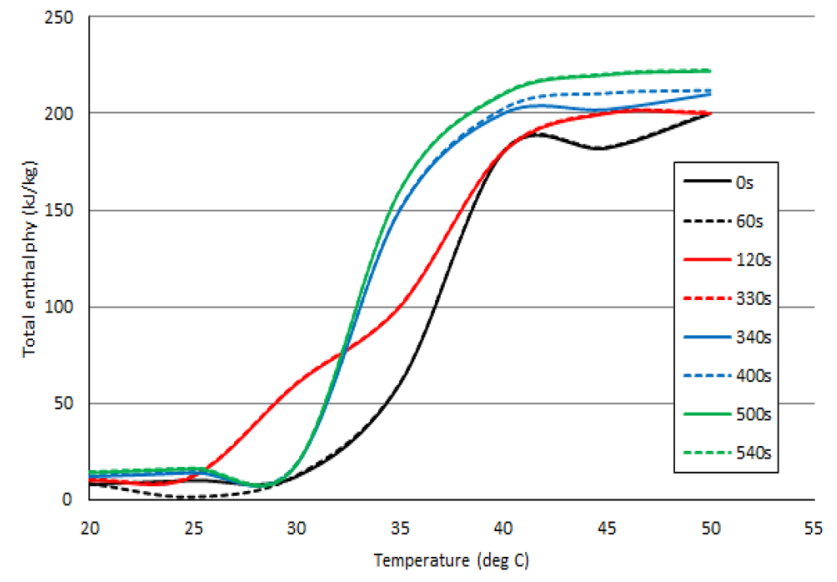

Fig. 9 Total enthalpy variation with respect to increasing temperature

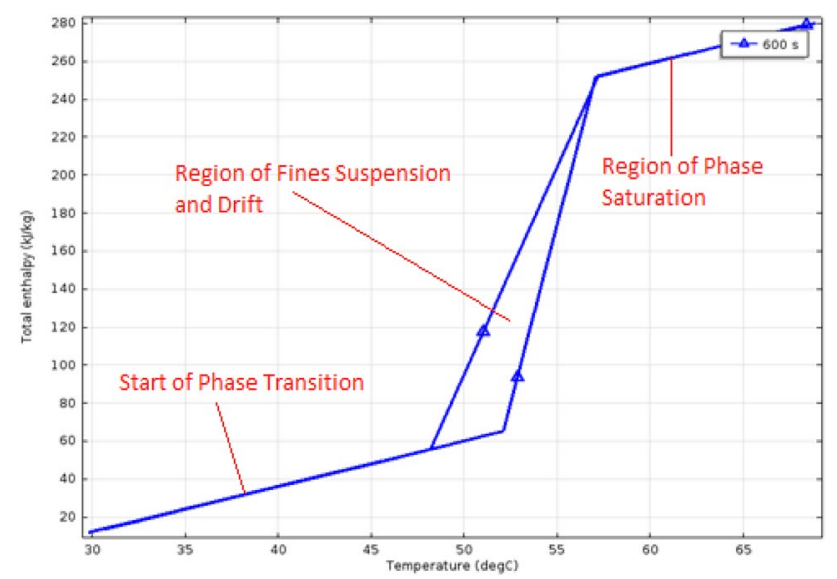

Fig. 10 Total enthalpy variation with respect to increasing temperature: $600 \mathrm{~s}$ of phase transition time

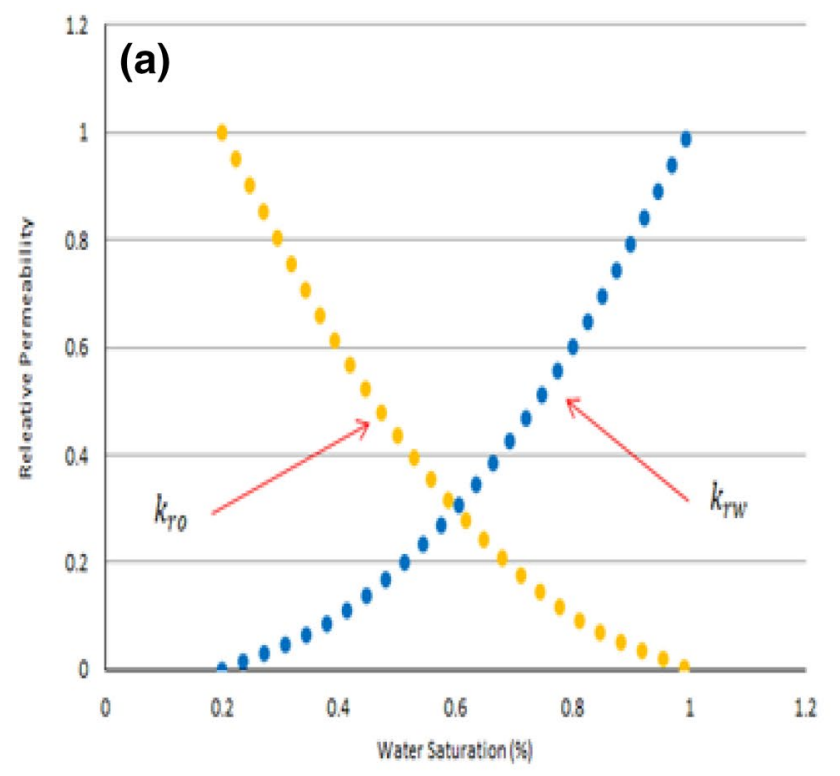

Fig. 11 Relative permeability curves: $\mathbf{a} \frac{k_{\mathrm{ro}}}{k_{\mathrm{rw}}}, \mathbf{b} \frac{k_{\mathrm{row}}}{k_{\mathrm{rg}}}$ mentioning the $600 \mathrm{~s}$ of phase transition time. It can be viewed from Fig. 10 that in the beginning, the fluid phase transition process rises steadily for gradual increase in the temperature. After some time, between $47^{\circ} \mathrm{C}$ and $53^{\circ} \mathrm{C}$ the curve climbs exponentially. Two curves were noted at these temperatures, and in the boundary region between this area is the regime of fines suspension and drift. During the phase transition that is while the oil is releasing the gas, the fines that are suspended in oil and water phases rises to the gas phase. Presently, the suspended fines will experience a two degrees of freedom that is the fine particles will undergo a collision with upper and lower pore wall, mostly with the upper pore wall and minima in the lower pore wall since the density of the oil-water phase is high. The suspended fines will drift in the pore space, and after completion of fluid phase saturation, the suspended concentration of fines and gas-liquid phase will be stagnated. Obviously, there will be a considerable quantity of heat release during phase transition, but in this case it will not alter the surface energy of the porous medium, fluid saturation, and fines migration as well.

Figure 11 shows the curves for the relative permeability of oil to water phase and oil-water phase to gas-liquid phase. It can be seen from Fig. 11a that increasing water saturation decreases the relative permeability of oil. Before oil production, the reservoir is only saturated with oil and we assume that there is no aquifer beneath the oil layer. Hence, the porosity of reservoir rocks and its permeability are not affected in anyway. But, during the secondary production process, that is when using the waterflood method to mobilize the oil, the water may travel ahead of the oil phase or it can be called as a water displacement front, leaving the oil phase behind. So with the rise in water saturation, the large

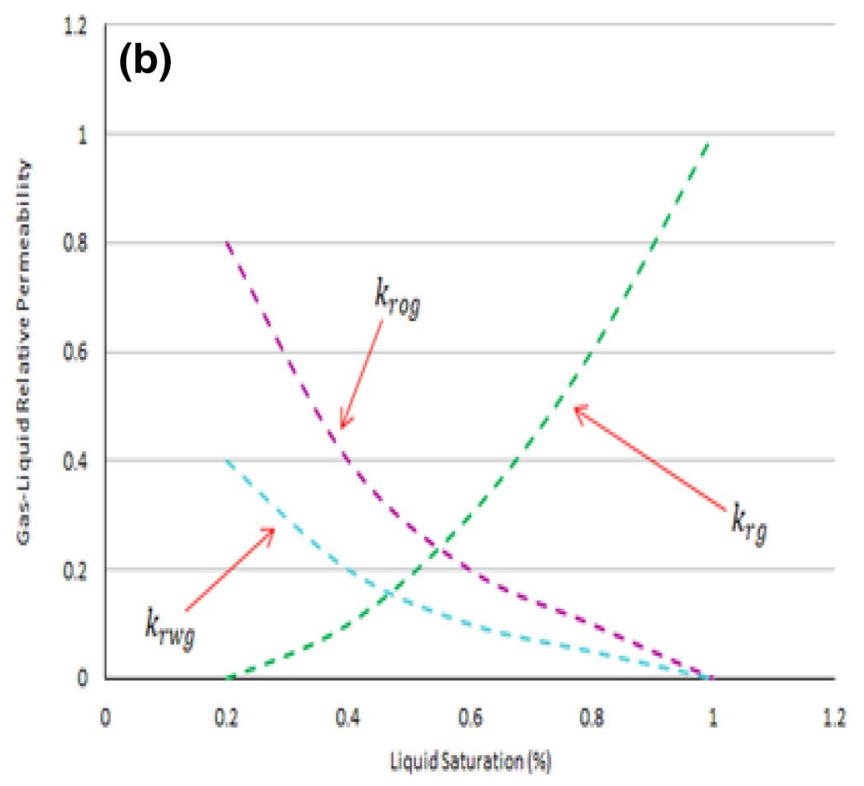




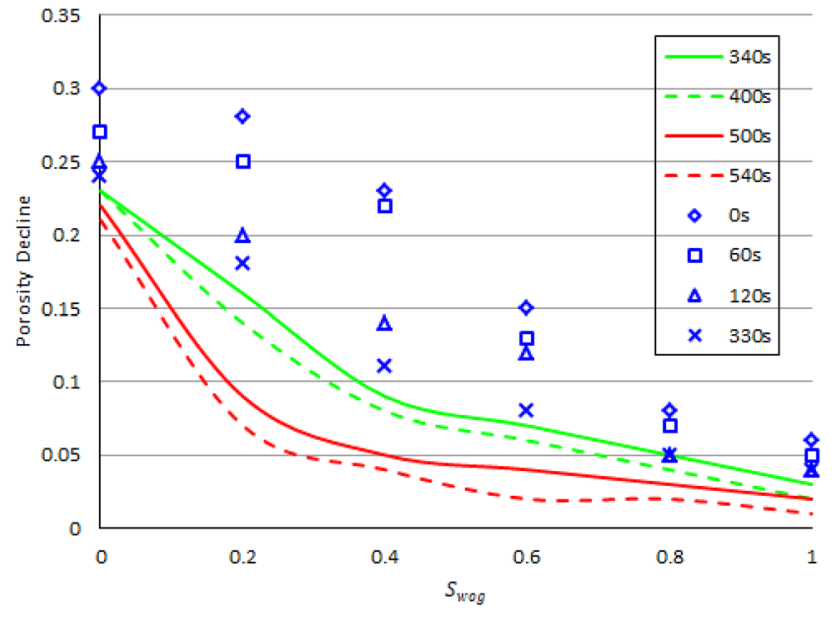

Fig. 12 Decreasing rock porosity for increasing $S_{\text {wog }}$

deposits of oil may not be effectively recovered and well may experience a water coning problem. Also, this phenomenon may be due to the consequences of the drawdown pressure. It can be observed from Fig. $11 \mathrm{~b}$ that the relative permeability of oil-gas phase and water-gas phase decreases for the rise in the newly created gas phase relative permeability. This gas release from the oil phase enhances the density of the overall fluid saturation and occupies the full surface energy of the porous media. Due to differences in fluid density and viscosity, the fluid will remain static and only sluggish fluid and fine particle movement can be observed.

Figure 12 shows the rock porosity decline for enhancing water-oil-gas saturation with respect to phase transition time. Initially, the porous reservoir was saturated with oil and water that gained half of porous medium surface energy. But, during the RV process a new phase was created by the reservoir, thereby exploiting the full surface energy of the pore chamber or in other words a high amount of fluid saturation in porous media decreases the available space in that medium and consequently, there will be an imbalance in the fluid $(W>O>\mathrm{G})$ density. As a result reservoir fluid will undergo a state of stagnation, where there will not be any fluid movement in the porous path. Moreover, increasing saturation can decrease the porosity and permeability of the reservoir rocks. As it is evident from Fig. 12 that at no multi-component fluid saturation the porosity of the reservoir rocks is noted between 0.2 and 0.3 . After a gradual increase in the saturation of three-phase fluids, the porosity decreases steadily reaching the lowest value below 0.05 . It can be observed that the porosity decline is higher for the $540 \mathrm{~s}$ phase transition time and lesser for zero seconds. Implying, that the rock porosity plummet sharply for higher phase transition time.

It can be seen from Fig. 13 that there is a close correlation between numerical, mathematical, and experimental

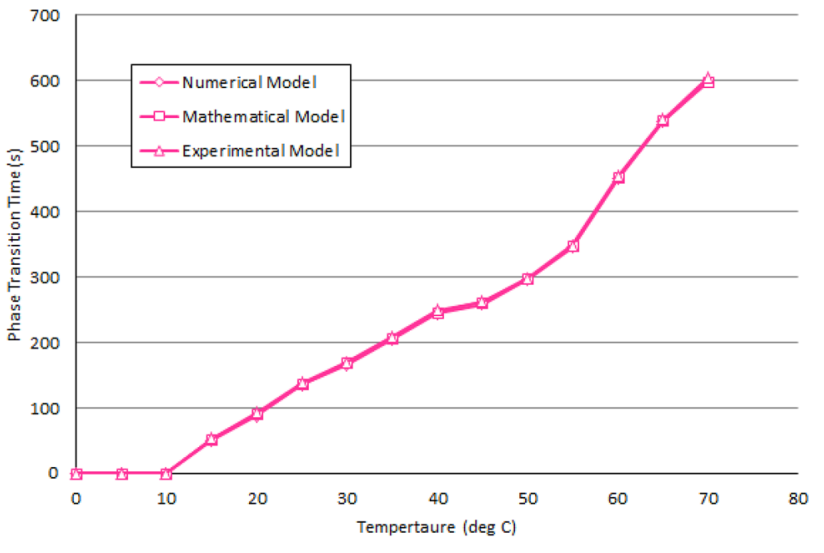

Fig. 13 Model validation for an oil reservoir which exhibits a retrograde vaporization behavior

models. The correlation was obtained by plotting phase transition time and temperature. Major factors such as density, velocity, viscosity, heat transfer rate, and mass flow rate were taken for the calculation. On then whole, all models revealed good agreement and there were no major differences between the model accuracies. Hence, it can be stated that this model is reliable for laboratory and field investigations on the reservoir formation damage due to fines migration in oil reservoirs which undergoes a rapid retrograde vaporization process. Therefore, this paper has successfully demonstrated the fines migration and drifting mechanisms due to colloidal forces of liquid and gas phases.

\section{Conclusions}

The first and foremost is that this paper has successfully demonstrated the phenomenon of retrograde vaporization in oil reservoirs and its subsequent impact on fines migration is also studied. Based on the mathematical and numerical modeling following conclusions can be drawn:

(1) A new equation for perturbations in the porous medium energy change was obtained by combining the pore surface fines mass balance and general energy equations. This has led to the creation of the fines energy conversion equation. We have demonstrated and validated this equation based on theoretical and numerical modeling.

(2) Retrograde vaporization simulation revealed that there was a tremendous amount of heat release during the fluid phase change process. This heat release and saturation detach and suspend the fines in the three-phase fluids. Consequently, it will roll and collide within the pore walls and drift along with the liquid-gas flow. Importantly, fines degrees of freedom are gradually getting arrested due to fluid phase density difference. 
(3) During fluid phase change heat transfer, there is an increase in the enthalpy of a new phase for the elevated reservoir temperature. The fines drift was identified in the region between the start of phase transition and saturation. The relative permeability of oil decreases with increasing saturation and also, relative permeability of oil-gas phase and water-gas phase decreases for increasing gas relative permeability. In addition, rock porosity decreases for increasing water-oil-gas saturation with respect to increasing phase transition time. Finally, this work is validated with experimental modeling and revealed good agreement.

(4) Therefore, the quantification and analysis of formation damage due to fines migration in oil reservoirs that undergo two- to three-phase flow is critical and important for optimizing the production loss. Initially, these factors must be seriously taken into consideration before the start of water injection. The experimental study of RV phenomenon and its interaction with low to high salinity water injection will be explored in our future work. Furthermore, our future work will also explore the RV effects on the EOR/IOR performances due to fines migration and it was already reported that fines migration can increase the water-oil relative permeability in porous rocks and expand the rock surface energy, which ultimately increase the mobility ratio and oil recovery rate (Hussain et al. 2013; Zeinijahromi et al. 2011). Therefore, in the near future, we will make numerical and thermal investigations on the effects of retrograde vaporization on the enhanced or improved oil recovery rate by taking natural reservoir fines into an account.

Acknowledgements The corresponding authors thank the management of Sathyabama Institute of Science and Technology for their financial and technical assistances.

Open Access This article is distributed under the terms of the Creative Commons Attribution 4.0 International License (http://creativeco mmons.org/licenses/by/4.0/), which permits unrestricted use, distribution, and reproduction in any medium, provided you give appropriate credit to the original author(s) and the source, provide a link to the Creative Commons license, and indicate if changes were made.

\section{References}

Abbaspour A, Hassanabad M (2010) Comparing sloshing phenomena in a rectangular container with and without a porous medium

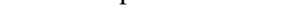

using explicit nonlinear 2-D BEM-FDM. Trans B Mech Eng 17:93-101

Alhuraishawy A, Bai AK, Wei M, Geng J, Pu J (2018) Mineral dissolution and fine migraton effect on oil recovery factor by lowsalinity water flooding in low-permeability sandstone reservoir. Fuel 220:898-907

Bashtani F, Ayatollahi S, Habibi A, Masihi M (2013) Permeability reduction of membranes during particulate suspension flow; analytical micro model of size exclusion mechanism. J Membr Sci 435:155-164

Chequer L, Vaz A, Bedrikovetsky P (2018) Injectivity decline during low salinity waterflooding due to fines migration. J Pet Sci Eng 165:1054-1072

Dullien F (1992) Porous media: fluid transport and pore structure, 2nd edn. Academic Press, San Diego, pp 35-75

Greenspan D (2005) A particle model of fluid sloshing. Math Comput Model 41:749-757

Hussain F, Zeinijahromi A, Bedrikovetsky P, Badalyan A, Carageorgos T, Cinar Y (2013) An experimental study of improved oil recovery through fines-assisted waterflooding. J Pet Sci Eng 109:187-197

Kampel G (2007) Mathematical modeling of fines migration and clogging in porous media. Georgia Institute of Technology, Atlanta

Khilar K, Fogler H (1998) Migrations of fines in porous media, 1 st edn. Kluwer Academic Publishers, Dordrecht. ISBN 978-90-481-5115-8

Oliveira M, Vaz A, Siqueira F, Yang Y, You Z, Bedrikovetsky P (2014) Slow migration of mobilised fines during flow in reservoir rocks: laboratory study. J Pet Sci Eng 122:534-541

Raha S, Khilar K, Kanpur P (2007) Regularities in pressure filtration of fine and colloidal suspensions. Int $\mathrm{J}$ Miner Process 84(1-4):348-360

Robinson B (1993) Production problems. In: Thompson D, Woods A (eds) Development geology reference manual. AAPG, Tulsa, pp 492-495

Santos A, Bedrikovetsky P, Fontoura A (2008) Analytical micro model for size exclusion: pore blocking and permeability reduction. $\mathrm{J}$ Membr Sci 308:115-127

Tistory (2018) What is waterflooding? (Water Injection). Plant Eng. http://plant-engineering.tistory.com/267

Yang Y, Siqueira F, Vaz A, You Z, Bedrikovetsky P (2016) Slow migration of detached fine particles over rock surface in porous media. J Nat Gas Sci Eng 34:1159-1173

You Z, Yang Y, Badalyan A, Bedrikovetsky P, Hand M (2016) Mathematical modelling of fines migration in geothermal reservoirs. Geothermics 59:123-133

Zeinijahromi A, Lemon P, Bedrikovetsky P (2011) Effects of induced fines migration on water cut during waterflooding. J Pet Sci Eng 78(3-4):609-617

Zeinijahromi A, Farajzadeh R, Bruining J, Bedrikovetsky P (2016) Effect of fines migration on oil-water relative permeability during two-phase flow in porous media. Fuel 176:222-236

Publisher's Note Springer Nature remains neutral with regard to jurisdictional claims in published maps and institutional affiliations. 